\title{
A FIXED POINT THEOREM FOR ANALYTIC FUNCTIONS
}

VALENTIN MATACHE

Received 4 August 2004

We prove that each analytic self-map of the open unit disk which interpolates between certain $n$-tuples must have a fixed point.

\section{Introduction}

Let $\mathbb{U}$ denote the open unit disk centered at the origin and $\mathbb{T}$ its boundary. For any pair of distinct complex numbers $z$ and $w$ and any positive constant $k$, we consider the locus of all points $\zeta$ in the complex plane $\mathbb{C}$ having the ratio of the distances to $w$ and $z$ equal to $k$, that is, we consider the solution set of the equation

$$
\frac{|\zeta-w|}{|\zeta-z|}=k
$$

We denote that set by $A(z, w, k)$ and (following [1]) call it the Apollonius circle of constant $k$ associated to the points $z$ and $w$. The set $A(z, w, k)$ is a circle for all values of $k$ other than 1 when it is a line.

In this paper, we consider $z, w \in \mathbb{U}$, show that if $z \neq w$, then necessarily $A(z, w$, $\left.\sqrt{\left(1-|w|^{2}\right) /\left(1-|z|^{2}\right)}\right)$ meets the unit circle twice, consider the arc on the unit circle with those endpoints, situated in the same connected component of $\mathbb{C} \backslash A(z, w$, $\left.\sqrt{\left(1-|w|^{2}\right) /\left(1-|z|^{2}\right)}\right)$ as $z$, and denote it by $\Gamma_{z, w}$. We prove that if $Z=\left(z_{1}, \ldots, z_{N}\right)$ and $W=\left(w_{1}, \ldots, w_{N}\right)$ are $N$-tuples with entries in $\mathbb{U}$ such that $z_{j} \neq w_{j}$ for all $j=1, \ldots, N$ and

$$
\mathbb{T}=\bigcup_{j=1}^{N} \Gamma_{z_{j}, w_{j}},
$$

then each analytic self-map of $\mathbb{U}$ interpolating between $Z$ and $W$ must have a fixed point. The next section contains the announced fixed point theorem (Theorem 2.2). 


\section{The fixed point theorem}

For each $e^{i \theta} \in \mathbb{T}$ and $k>0$, the set

$$
\operatorname{HD}\left(e^{i \theta}, k\right):=\left\{z \in \mathbb{U}:\left|e^{i \theta}-z\right|^{2}<k\left(1-|z|^{2}\right)\right\}
$$

called the horodisk with constant $k$ tangent at $e^{i \theta}$ is an open disk internally tangent to $\mathbb{T}$ at $e^{i \theta}$ whose boundary $\operatorname{HC}\left(e^{i \theta}, k\right):=\left\{z \in \mathbb{U}:\left|e^{i \theta}-z\right|^{2}=k\left(1-|z|^{2}\right)\right\}$ is called the horocycle with constant $k$ tangent at $e^{i \theta}$.

The center and radius of $\mathrm{HC}\left(e^{i \theta}, k\right)$ are given by

$$
C=\frac{e^{i \theta}}{1+k}, \quad R=\frac{k}{1+k},
$$

respectively. One should note that $\operatorname{HD}\left(e^{i \theta}, k\right)$ extends to exhaust $\mathbb{U}$ as $k \rightarrow \infty$.

Let $\varphi$ be a self-map of $\mathbb{U}$. For each positive integer $n, \varphi^{[n]}=\varphi \circ \varphi \circ \cdots \circ \varphi, n$ times. The following is a combination of results due to Denjoy, Julia, and Wolff.

Theorem 2.1. Let $\varphi$ be an analytic self-map of $\mathbb{U}$. If $\varphi$ has no fixed point, then there is a remarkable point $w$ on the unit circle such that the sequence $\left\{\varphi^{[n]}\right\}$ converges to $w$ uniformly on compact subsets of $\mathbb{U}$ and

$$
\varphi(\operatorname{HD}(w, k)) \subseteq \operatorname{HD}(w, k) \quad k>0 .
$$

The remarkable point $w$ is called the Denjoy-Wolff point of $\varphi$. Relation (2.3) is a consequence of a geometric function-theoretic result known as Julia's lemma. In case $\varphi$ has a fixed point, but is not the identity or an elliptic disk automorphism, one can use Schwarz's lemma in classical complex analysis to show that $\left\{\varphi^{[n]}\right\}$ tends to that fixed point, (which is also regarded as a constant function), uniformly on compact subsets of $\mathbb{U}$. These facts show that if $\varphi$ is not the identity, then it may have at most a fixed point in $\mathbb{U}$. Good accounts on all the results summarized above can be found in [2, Section 2.3] and [4, Sections 4.4-5.3].

In the sequel, $\varphi$ will always denote an analytic self-map of $\mathbb{U}$ other than the identity. For each $z \in \mathbb{U}$ such that $\varphi(z) \neq z$, we consider the intersection of the unit circle $\mathbb{T}$ and $A\left(z, \varphi(z), \sqrt{\left(1-|\varphi(z)|^{2}\right) /\left(1-|z|^{2}\right)}\right)$. It necessarily consists of two points.

Indeed, it cannot be a singleton. If one assumes that the aforementioned intersection is the singleton $\left\{e^{i \theta}\right\}$, then the relation

$$
\frac{\left|e^{i \theta}-\varphi(z)\right|^{2}}{1-|\varphi(z)|^{2}}=\frac{\left|e^{i \theta}-z\right|^{2}}{1-|z|^{2}}
$$

must be satisfied, and this means that both $z$ and $\varphi(z)$ are on a horocycle tangent to $\mathbb{T}$ at $e^{i \theta}$, which is contradictory due to the fact of, under our assumptions, $A(z, \varphi(z)$, $\left.\sqrt{\left(1-|\varphi(z)|^{2}\right) /\left(1-|z|^{2}\right)}\right)$ is also such a horocycle and hence fails to separate $z$ and $\varphi(z)$ (the points $z$ and $\varphi(z)$ should be in different connected components of $\mathbb{C} \backslash A(z, \varphi(z)$, $\left.\left.\sqrt{\left(1-|\varphi(z)|^{2}\right) /\left(1-|z|^{2}\right)}\right)\right)$. 
On the other hand, $\mathbb{T} \cap A\left(z, \varphi(z), \sqrt{\left(1-|\varphi(z)|^{2}\right) /\left(1-|z|^{2}\right)}\right)$ cannot be empty. Indeed, for any $z, w \in \mathbb{U}, z \neq w, A\left(z, w, \sqrt{\left(1-|w|^{2}\right) /\left(1-|z|^{2}\right)}\right)$ meets $\mathbb{T}$. To prove that, one can assume without loss of generality that $\left(1-|w|^{2}\right) /\left(1-|z|^{2}\right)>1$. If, arguing by contradiction, we assume that $A\left(z, w, \sqrt{\left(1-|w|^{2}\right) /\left(1-|z|^{2}\right)}\right) \cap \mathbb{T}=\varnothing$, then $\mathbb{T}$ must be exterior to $A\left(z, w,\left(1-|w|^{2}\right) /\left(1-|z|^{2}\right)\right)$, that is,

$$
\left|\frac{e^{i \theta}-w}{e^{i \theta}-z}\right|^{2}<\frac{1-|w|^{2}}{1-|z|^{2}} \quad \text { or, equivalently, } \quad \frac{\left|e^{i \theta}-w\right|^{2}}{1-|w|^{2}}<\frac{\left|e^{i \theta}-z\right|^{2}}{1-|z|^{2}} \quad e^{i \theta} \in \mathbb{T} .
$$

The last inequality implies that, for each $e^{i \theta} \in \mathbb{T}, w$ is interior to the horocycle $H$ tangent to $\mathbb{T}$ at $e^{i \theta}$ that passes through $z$. This leads to a contradiction since there exist horocycles that are exteriorly tangent to each other at $z$.

Thus $\mathbb{R} \cap A\left(z, \varphi(z), \sqrt{\left(1-|\varphi(z)|^{2}\right) /\left(1-|z|^{2}\right)}\right)$ necessarily consists of two points. Let $\Gamma_{z, \varphi(z)}$ denote the open arc of $\mathbb{T}$ with those endpoints, situated in the same connected component of $\mathbb{C} \backslash A\left(z, \varphi(z), \sqrt{\left(1-|\varphi(z)|^{2}\right) /\left(1-|z|^{2}\right)}\right)$ as $z$.

By straightforward computations, one can obtain the following formulas for the endpoints $e^{i \theta_{1}}$ and $e^{i \theta_{2}}$ of $\Gamma_{z, \varphi(z)}$ :

$$
e^{i \theta_{1,2}}=\frac{-\mu \pm i \sqrt{|\Lambda|^{2}-\mu^{2}}}{\Lambda}
$$

where

$$
\Lambda=\bar{z}\left(1-|\varphi(z)|^{2}\right)-\overline{\varphi(z)}\left(1-|z|^{2}\right), \quad \mu=|\varphi(z)|^{2}-|z|^{2}
$$

It is always true that $\Lambda \neq 0$ and $|\Lambda|>|\mu|$, as the reader can readily check.

We are now ready to state and prove the main result of this mathematical note.

Theorem 2.2. If there exist $z_{1}, z_{2}, \ldots, z_{N}$ such that $\varphi\left(z_{j}\right) \neq z_{j}, j=1, \ldots, N$, and

$$
\mathbb{T}=\bigcup_{j=1}^{N} \Gamma_{z_{j}, \varphi\left(z_{j}\right)}
$$

then $\varphi$ has a fixed point in $\mathbb{U}$. In particular, if $z_{1}, z_{2}, \ldots, z_{N} \in \mathbb{C} \backslash\{0\}$ are zeros of $\varphi$ and

$$
\mathbb{T}=\bigcup_{j=1}^{N}\left\{e^{i \theta}:\left|\theta-\arg \left(z_{j}\right)\right|<\arccos \left|z_{j}\right|\right\},
$$

then $\varphi$ has a fixed point in $\mathbb{U}$. Conversely, if $\varphi$ is an analytic self-map of $\mathbb{U}$ other than the identity and $\varphi$ has a fixed point, then there exist finitely many points $z_{1}, \ldots, z_{k}$ in $\mathbb{U}$ such that condition (2.8) is satisfied.

Proof. Observe that if $e^{i \theta} \in \Gamma_{z, \varphi(z)}$, then $e^{i \theta}$ cannot be the Denjoy-Wolff point of $\varphi$. Indeed, arguing by contradiction, assume $e^{i \theta}$ is the Denjoy-Wolff point of $\varphi$. Note that one can consider a horodisk $\operatorname{HD}\left(e^{i \theta}, k\right)$ for which $z$ is interior and $\varphi(z)$ exterior, since $\left|e^{i \theta}-z\right|^{2} /\left(1-|z|^{2}\right)<\left|e^{i \theta}-\varphi(z)\right|^{2} /\left(1-|\varphi(z)|^{2}\right)$. This leads to a contradiction by (2.3). 


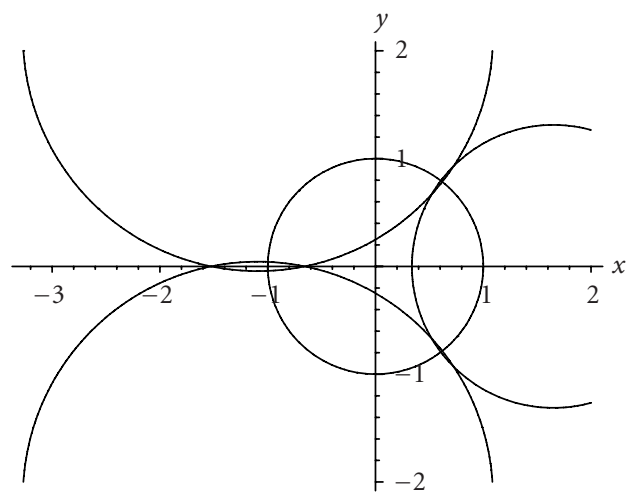

Figure 2.1

Thus if (2.8) holds, then $\varphi$ does not have a Denjoy-Wolff point, that is, it has a fixed point in $\mathbb{U}$. Finally, observe that if $z \neq 0$ and $\varphi(z)=0$, a simple computation leads to $\Gamma_{z, \varphi(z)}=\left\{e^{i \theta}:|\theta-\arg (z)|<\arccos |z|\right\}$, which takes care of (2.9).

To prove the necessity of condition (2.8) now, assume $\varphi$ is not the identity and has a fixed point $\omega \in \mathbb{U}$. Let $\rho(z, w):=|z-w| /|1-\bar{w} z|, z, w \in \mathbb{U}$, denote the pseudohyperbolic distance on $\mathbb{U}$. For each $z_{0} \in \mathbb{U}$ and $r>0$, let $K\left(z_{0}, r\right):=\left\{z \in \mathbb{U}: \rho\left(z, z_{0}\right)<r\right\}$ be the pseudohyperbolic disk of center $z_{0}$ and radius $r$. Pseudohyperbolic disks are also Euclidean disks inside $\mathbb{U}$ (see [3, page 3]), and if $r<1$, then $K\left(z_{0}, r\right) \neq \mathbb{U}$. By the invariant Schwarz lemma, (see [3, Lemma 1.2]), one has that $\rho(\varphi(z), \omega) \leq \rho(z, \omega), z \in \mathbb{U}$. This means that $\varphi$ maps closed pseudohyperbolic disks with pseudohyperbolic center $\omega$ into themselves. We record this fact for later use and proceed by noting that condition (2.8) is satisfied for some finite set of points in $\mathbb{U}$ if and only if

$$
\mathbb{T}=\bigcup_{z \in \mathbb{U} \backslash\{\omega\}} \Gamma_{z, \varphi(z)}
$$

which is a direct consequence of the compactness of $\mathbb{T}$. Thus, arguing by contradiction, one should assume that there exists $e^{i \theta} \in \mathbb{T}$ such that, for each $z \neq \omega$, one has that $e^{i \theta} \notin$ $\Gamma_{z, \varphi(z)}$, that is, $\left|e^{i \theta}-z\right|^{2} /\left(1-|z|^{2}\right)>\left|e^{i \theta}-\varphi(z)\right|^{2} /\left(1-|\varphi(z)|^{2}\right)$. One deduces that, for each $z \neq \omega, \varphi(z)$ is interior to the horocycle $H$ tangent to $\mathbb{T}$ at $e^{i \theta}$ that passes through $z$. This generates a contradiction. Indeed, consider some $0<r<1$ and the pseudohyperbolic disk $K(\omega, r)$. Let $H$ be the horocycle tangent at $e^{i \theta}$ to $\mathbb{T}$ which is also exteriorly tangent to $\partial K(\omega, r)$. Denote this tangence point by $z$. Since $\omega \in K(\omega, r), z \neq \omega$. On the other hand, it is impossible that $\varphi(z)$ be simultaneously interior to $H$ and in the closure of $K(\omega, r)$.

Example 2.3. Any holomorphic self-map of $\mathbb{U}$ interpolating between the triples $(0.34,0.5 i$, $-0.5 i)$ and $(0.335,0.25+0.125 i, 0.25-0.125 i)$ has a fixed point in $\mathbb{U}$, because

$$
\mathbb{T}=\Gamma_{0.34,0.335} \cup \Gamma_{0.5 i, 0.25+0.125 i} \cup \Gamma_{-0.5 i, 0.25-0.125 i}
$$


as one can readily check by using relations (2.6) and (2.7) (see also Figure 2.1 which illustrates the equality above). The fact that such holomorphic self-maps exist can be checked by using Pick's interpolation theorem, (see [3, Theorem 2.2]) or (much easier) by noting that $\varphi(z)=(z+1) / 4$ is such a map.

\section{References}

[1] L. V. Ahlfors, Complex Analysis, 3rd ed., McGraw-Hill, New York, 1978.

[2] C. C. Cowen and B. D. MacCluer, Composition Operators on Spaces of Analytic Functions, Studies in Advanced Mathematics, CRC Press, Florida, 1995.

[3] J. B. Garnett, Bounded Analytic Functions, Pure and Applied Mathematics, vol. 96, Academic Press, New York, 1981.

[4] J. H. Shapiro, Composition Operators and Classical Function Theory, Universitext: Tracts in Mathematics, Springer-Verlag, New York, 1993.

Valentin Matache: Department of Mathematics, University of Nebraska, Omaha, NE 68182, USA E-mail address: vmatache@mail.unomaha.edu 